\title{
FEW REMARKS ON EVOLUTION ALGEBRAS
}

\author{
ABROR KH. KHUDOYBERDIYEV, BAKHROM A. OMIROV, IZZAT QARALLEH
}

\begin{abstract}
In the present paper we study some algebraic properties of evolution algebras. Moreover, we reduce the study of evolution algebras of permutations to two special types of evolution algebras, idempotents and absolute nilpotent elements of the algebra. We study three-dimensional evolution algebras whose each element of evolution basis has infinite period. In addition, for an evolution algebra with some properties we describe its associative enveloping algebra.
\end{abstract}

Mathematics Subject Classification 2010: 17D92, 17D99.

Key Words and Phrases: evolution algebra, algebra of permutations, absolute nilpotent element, idempotent, algebra of multiplications, associative enveloping algebra.

\section{INTRODUCTION.}

In 20 s and 30 s of the last century the new object was introduced to mathematics, which was the product of interactions between Mendelian genetics and mathematics. One of the first scientist who give an algebraic interpretation of the " $\times$ " sign, which indicated sexual reproduction was Serebrowsky.

It is known that there exists an intrinsic and general mathematical structure behind the neutral Wright-Fisher models in population genetics, the reproduction of bacteria involved by bacteriophages, asexual reproduction or generally non-Mendelian inheritance and Markov chains. In 10] a new type of algebras was associated with it - the evolution algebras.

Although an evolution algebra is an abstract system, it gives an insight for the study of nonMendelian genetics. For instance, an evolution algebra can be applied to the inheritance of organelle genes, one can predict, in particular, all possible mechanisms to establish the homoplasmy of cell populations.

The general genetic algebras developed into a field of independent mathematical interest, because these algebras are in general non-associative and do not belong to any of the well-known classes of non-associative algebras such as Lie algebras, alternative algebras, or Jordan algebras.

Until 1980s, the most comprehensive reference in this area was Wörz-Busekros's book [11. More recent results, such as genetic evolution in genetic algebras, can be found in Lyubich's book [7. A good survey is Reed's article [8. In Tian's book [10] a foundation of the framework of the theory of evolution algebras is established and some applications of evolution algebras in the theory of stochastic processes and genetics are discussed. Recently, Rozikov and Tian [9] studied algebraic structures evolution algebras associated with Gibbs measures defined on some graphs. In [2], [5], 6] derivations, some properties of chain of evolution algebras and dibaricity of evolution algebras were studied. In [1, 3, 4] certain algebraic properties of evolution algebras (like right nilpotency, nilpotency and solvability etc.) in terms of matrix of structural constants have been investigated. In fact, nilpotency, right nilpotency and solvability might be interpreted in a biological way as a various types of vanishing ("deaths") populations.

The present paper is organized as follows: In Section 2 we give some definitions and preliminary results. In Section 3 we reduce the study of arbitrary evolution algebra of permutations into two special evolution algebras. Section 4 is devoted to the description of $n$-dimensional associative enveloping algebras of $n$-dimensional evolution algebras with some restrictions on rank of the matrix $A$ of structural constants. Moreover, associative enveloping algebras for 2-dimensional evolution algebras are described, as well. In Section 5 we establish some properties of three-dimensional evolution algebras whose each basis element has infinite period.

Throughout the paper we consider finite-dimensional evolution algebras over a field of zero characteristic. Moreover, in the multiplication table of an evolution algebra the omitted products are assumed to be zero.

\section{Preliminaries.}

Let us define the main object of this work - evolution algebra. 
Definition 2.1. [10] Let $(E, \cdot)$ be an algebra over a field $F$. If it admits a basis $\left\{e_{1}, e_{2}, \ldots\right\}$ such that

$$
e_{i} \cdot e_{j}=0, \quad \text { for } i \neq j, \quad e_{i} \cdot e_{i}=\sum_{k} a_{i, k} e_{k}, \quad \text { for any } i,
$$

then this algebra is called evolution algebra.

It is remarkable that this type of algebra depends on evolution basis $\left\{e_{1}, e_{2}, \ldots\right\}$.

In the following theorem we present the list (up to isomorphism) of 2-dimensional complex evolution algebras.

Theorem 2.2. 4 Any 2-dimensional non abelian complex evolution algebra $E$ is isomorphic to one of the following pairwise non isomorphic algebras:

(1) $\operatorname{dim} E^{2}=1$

- $E_{1}: e_{1} e_{1}=e_{1}$,

- $E_{2}: e_{1} e_{1}=e_{1}, \quad e_{2} e_{2}=e_{1}$,

- $E_{3}: e_{1} e_{1}=e_{1}+e_{2}, \quad e_{2} e_{2}=-e_{1}-e_{2}$,

- $E_{4}: e_{1} e_{1}=e_{2}$.

(2) $\operatorname{dim} E^{2}=2$

- $E_{5}: \quad e_{1} e_{1}=e_{1}+a_{2} e_{2}, \quad e_{2} e_{2}=a_{3} e_{1}+e_{2}, \quad 1-a_{2} a_{3} \neq 0$, where $E_{5}\left(a_{2}, a_{3}\right) \cong E_{5}^{\prime}\left(a_{3}, a_{2}\right)$,

- $E_{6}: e_{1} e_{1}=e_{2}, \quad e_{2} e_{2}=e_{1}+a_{4} e_{2}$, where for $a_{4} \neq 0, E_{6}\left(a_{4}\right) \cong E_{6}\left(a_{4}^{\prime}\right) \Leftrightarrow \frac{a_{4}^{\prime}}{a_{4}}=$ $\cos \frac{2 \pi k}{3}+i \sin \frac{2 \pi k}{3}$ for some $k=0,1,2$.

Further we shall show the role of idempotents and absolute nilpotent elements of an evolution algebra.

Definition 2.3. An element $x$ of an evolution algebra $E$ is called idempotent, if $x x=x$. An element $y$ of an evolution algebra $E$ is called absolute nilpotent if $y y=0$.

Consider a complex evolution algebra $E_{n, \pi}\left(a_{1}, a_{2}, \ldots, a_{n}\right)$ with a basis $\left\{e_{1}, e_{2}, \ldots, e_{n}\right\}$ and the table of multiplications given by

$$
\begin{cases}e_{i} \cdot e_{i}=a_{i} e_{\pi(i)}, & 1 \leq i \leq n \\ e_{i} \cdot e_{j}=0, & i \neq j,\end{cases}
$$

where $\pi$ is an element of the group of permutations $S_{n}$.

An evolution algebra $E_{n, \pi}\left(a_{1}, a_{2}, \ldots, a_{n}\right)$ is said to be evolution algebra of permutations.

In what follows, by a cycle permutation we mean a permutation in which a part of symbols $\left\{l_{1}, l_{2}, \ldots, l_{t}\right\} \subseteq\{1,2, \ldots, n\}$ are cyclic permutated and the rest ones are stationary, i.e., $l_{1} \rightarrow l_{2} \rightarrow$ $\cdots \rightarrow l_{t} \rightarrow l_{1}$, and we denote $\pi=\left(l_{1}, l_{2}, \ldots, l_{t}\right)$.

It is known that any permutation is up to order uniquely decomposed into product of independent cycles.

For permutations of the form $\pi=\left(l_{1}, l_{2}, \ldots, l_{r}\right)\left(m_{1}, m_{2}, \ldots, m_{s}\right) \ldots\left(p_{1}, p_{2}, \ldots, p_{t}\right)$ it is known the following result.

Proposition 2.4. Two permutations are conjugated in $S_{n}$ if and only if the corresponding sets $\{r, s, \ldots, t\}$ are coincided.

Definition 2.5. 10] An evolution algebra $E$ with a table of multiplications

$$
e_{i} \cdot e_{i}=\sum_{k} a_{i, k} e_{k}, \quad a_{i} e_{j}=0, i \neq j
$$

is called Markov evolution algebra if $\sum_{k} a_{i, k}=1$.

For a given element $x$ of an evolution algebra $E$ we consider the right multiplication operator $R_{x}: E \rightarrow E$ defined by $R_{x}(y)=y x, y \in E$.

Note that operators of right and left multiplications are coincided, since evolution algebras are commutative.

For an evolution algebra $E$, by $M(E)$ we denote an associative enveloping algebra which is generated by the set $R(E)=\left\{R_{x} \mid x \in E\right\}$. It is clear that $M(E)$ is a subalgebra of $\operatorname{End}(E)$.

For an element $x \in E$ we define plenary powers as follows:

$$
x^{[1]}=x, \quad x^{[k+1]}=x^{[k]} \cdot x^{[k]}, \quad k \geq 1 .
$$


Definition 2.6. Let $e_{j}$ be a generator of an evolution algebra $E$, the period $d$ of $e_{j}$ is defined to be the greatest common divisor of the set $\left\{\log _{2} m \mid e_{j}<e_{j}^{[m]}\right\}$. That is

$$
d=\text { g.c.d. }\left\{\log _{2} m \mid e_{j}<e_{j}^{[m]}\right\} .
$$

\section{Evolution ALgEBra of PERMUtations}

Let us first present two important examples of evolution algebra of permutations.

Example 3.1. Consider the following evolution algebra:

$$
E_{n}:\left\{\begin{array}{l}
e_{i} \cdot e_{i}=e_{i+1}, \quad 1 \leq i \leq n-1, \\
e_{n} \cdot e_{n}=e_{1}, \\
e_{i} \cdot e_{j}=0, \quad i \neq j .
\end{array}\right.
$$

Evidently, the algebra $E_{n}$ is evolution algebra of permutations of the form $E_{n, \pi}(1,1, \ldots, 1)$, with $\pi=$ $(1,2,3, \ldots, n)$.

Example 3.2. Evolution algebra defined as follows:

$$
E N_{n}:\left\{\begin{array}{l}
e_{i} \cdot e_{i}=e_{i+1}, \quad 1 \leq i \leq n-1, \\
e_{n} \cdot e_{n}=0, \\
e_{i} \cdot e_{j}=0, \quad i \neq j,
\end{array}\right.
$$

is the algebra of permutations of the form $E_{n, \pi}(1,1, \ldots, 1,0)$ with $\pi=(1,2,3, \ldots, n)$.

Note that $E_{n}, E N_{n}$ are single-generated simple and nilpotent evolution algebras, respectively. Moreover, algebras $E_{1}, E N_{1}$ define one-dimensional evolution algebras, whose basis elements are idempotent and absolute nilpotent elements, respectively.

Now we shall consider some properties of evolution algebra of permutations.

Proposition 3.3. Let $E_{n, \pi}\left(a_{1}, a_{2}, \ldots, a_{n}\right)$ be an evolution algebra of permutations with the following conditions:

(i) $a_{i} \neq 0$ for all $i(1 \leq i \leq n)$,

(ii) $\pi=\pi_{1} \circ \pi_{2} \circ \cdots \circ \pi_{r}$, where $\pi_{1}=\left(l_{1}, l_{2}, \ldots, l_{k_{1}}\right), \quad \pi_{2}=\left(m_{1}, m_{2}, \ldots, m_{k_{2}}\right), \ldots, \quad \pi_{r}=$ $\left(p_{1}, p_{2}, \ldots, p_{k_{r}}\right)$ are independent cycles and $k_{1}+k_{2}+\cdots+k_{r}=n$.

Then

$$
E_{n, \pi}\left(a_{1}, a_{2}, \ldots, a_{n}\right) \cong E_{k_{1}, \pi_{1}}\left(b_{1}, b_{2}, \ldots, b_{k_{1}}\right) \oplus E_{k_{2}, \pi_{2}}\left(c_{1}, c_{2}, \ldots, c_{k_{2}}\right) \oplus \cdots \oplus E_{k_{r}, \pi_{r}}\left(d_{1}, d_{2}, \ldots, d_{k_{r}}\right) .
$$

Proof. The isomorphism is provided by the following change of basis:

$$
e_{i, 1}=e_{l_{i}}, 1 \leq i \leq k_{1}, \quad e_{i, 2}=e_{m_{i}}, 1 \leq i \leq k_{2}, \quad \ldots, \quad e_{i, r}=e_{p_{i}}, 1 \leq i \leq k_{r} .
$$

Thus, we have the evolution algebra $E_{k_{s}, \pi_{s}}(*, *, \ldots, *)$ with the basis $e_{i, s}, 1 \leq i \leq k_{s}, 1 \leq s \leq r$ and

$$
E_{n, \pi}\left(a_{1}, a_{2}, \ldots, a_{n}\right) \cong E_{k_{1}, \pi_{1}}\left(b_{1}, b_{2}, \ldots, b_{k_{1}}\right) \oplus E_{k_{2}, \pi_{2}}\left(c_{1}, c_{2}, \ldots, c_{k_{2}}\right) \oplus \cdots \oplus E_{k_{r}, \pi_{r}}\left(d_{1}, d_{2}, \ldots, d_{k_{r}}\right)
$$

for some non-zero values of $b_{i}, c_{j}, \ldots, d_{s}$.

In the following proposition we specify more details on the terms of direct sum in the statement of Proposition 3.3

Proposition 3.4. Any evolution algebra of permutation $E_{n, \tau}\left(a_{1}, a_{2}, \ldots, a_{n}\right)$ with $\tau=\left(l_{1}, l_{2}, \ldots, l_{n}\right)$ and condition $a_{i} \neq 0$ for all $i(1 \leq i \leq n)$ is isomorphic to the algebra $E_{n, \pi}\left(a_{1}, a_{\pi(1)}, \ldots, a_{\pi^{n-1}(1)}\right)$ with $\pi=(1,2, \ldots, n)$.

Proof. The isomorphism is established by basis permutation:

$$
e_{1}^{\prime}=e_{1}, \quad e_{i}^{\prime}=e_{\pi^{i-1}(1)}, 2 \leq i \leq n .
$$

Theorem 3.5. Any evolution algebra of permutation $E_{n, \tau}\left(a_{1}, a_{2}, \ldots, a_{n}\right)$ with $\tau=\left(l_{1}, l_{2}, \ldots, l_{n}\right)$ and condition $a_{i} \neq 0$ for all $i(1 \leq i \leq n)$ is isomorphic to the algebra $E_{n}$. 
Proof. Taking into account Proposition 3.4 it is sufficient to establish isomorphism between evolution algebra $E_{n, \pi}\left(a_{1}, a_{2}, \ldots, a_{n}\right)$ with $\pi=(1,2, \ldots, n)$ and non zero values of $a_{i}, 1 \leq i \leq n$ and evolution algebra $E_{n}$.

The application of the following scaling of basis:

$$
e_{i}^{\prime}=A_{i} e_{i}, 1 \leq i \leq n \quad \text { with } A_{i}=\sqrt[2^{n}-1]{\frac{1}{a_{i}^{2^{n-1}} a_{i+1}^{2^{n-2}} \ldots a_{n}^{2^{i-1}} a_{1}^{2^{i-2}} a_{2}^{2^{i-3}} \ldots a_{i-1}}},
$$

deduces products

$$
\left\{\begin{array}{l}
e_{i}^{\prime} \cdot e_{i}^{\prime}=e_{i+1}^{\prime}, \quad 1 \leq i \leq n-1, \\
e_{n}^{\prime} \cdot e_{n}^{\prime}=e_{1}^{\prime}, \\
e_{i}^{\prime} \cdot e_{j}^{\prime}=0, \quad i \neq j
\end{array}\right.
$$

Now we consider the case of $a_{i}=0$ for some $i \in\{1,2, \ldots, n\}$.

Proposition 3.6. Any evolution algebra of permutation $E_{n, \pi}\left(a_{1}, a_{2}, \ldots, a_{n}\right)$ with $\pi=\left(l_{1}, l_{2}, \ldots, l_{n}\right)$ and condition $a_{i}=0$ for some $i \in\{1,2, \ldots, n\}$ is isomorphic to the algebra $E N_{k_{1}} \oplus E N_{k_{2}} \oplus \cdots \oplus E N_{k_{r}}$.

Proof. Similarly to the proof of Proposition 3.4 taking the change

$$
e_{1}^{\prime}=e_{1}, \quad e_{i}^{\prime}=e_{\pi^{i-1}(1)}, 2 \leq i \leq n,
$$

we can suppose $\pi=(1,2, \ldots, n)$.

Let $a_{i_{1}}=a_{i_{2}}=\cdots=a_{i_{r}}=0$ for $i_{1}<i_{2}<\cdots<i_{r}$ and the rest are non-zero.

If $i_{r}=n$, then similarly as above we can assume that all $a_{i}=1$ for $1 \leq i \leq n-1$ and hence, $E_{n, \pi}\left(a_{1}, a_{2}, \ldots, a_{n}\right)$ with $\pi=(1,2, \ldots, n)$ is isomorphic to the algebra $E_{n}$.

If $i_{r}<n$, then taking the following change of basis:

$$
\begin{gathered}
e_{s}^{1}=e_{i_{r}+s}, 1 \leq s \leq n-i_{r}, \\
e_{n-i_{r}+s}^{1}=e_{s}, 1 \leq s \leq i_{1}, \\
e_{s}^{2}=e_{i_{1}+s}, 1 \leq s \leq i_{2}-i_{1}, \\
\ldots \quad \ldots \quad \ldots \quad \ldots \\
e_{s}^{r}=e_{i_{r-1}+s}, 1 \leq s \leq i_{r}-i_{r-1} .
\end{gathered}
$$

we obtain that $E_{n, \pi}\left(a_{1}, a_{2}, \ldots, a_{n}\right)$ with $\pi=(1,2, \ldots, n)$ is isomorphic to the algebra $E N_{k_{1}}\left(a_{1}, \ldots, a_{k_{1}-1}\right) \oplus E N_{k_{2}}\left(b_{1}, \ldots, b_{k_{2}-1}\right) \oplus \cdots \oplus E N_{k_{r}}\left(c_{1}, \ldots, c_{k_{r}-1}\right)$, where each of the algebra $E N_{k_{s}}(*, *, \ldots, *), 1 \leq s \leq r$ has the model of the following evolution algebra:

$$
E N_{k}\left(a_{1}, \ldots, a_{k-1}\right): \begin{cases}e_{i} \cdot e_{i}=a_{i} e_{i+1}, & 1 \leq i \leq k-1, a_{i} \neq 0, \\ e_{i} \cdot e_{j}=0, & i \neq j .\end{cases}
$$

Taking the basis transformation in the algebra $E N_{k}\left(a_{1}, \ldots, a_{k-1}\right)$ :

$$
e_{1}^{\prime}=e_{1}, e_{2}^{\prime}=a_{1} e_{2}, e_{3}^{\prime}=a_{1}^{2} a_{2} e_{3}, \ldots, e_{k}^{\prime}=a_{1}^{2^{k-2}} a_{2}^{2^{k-3}} \ldots a_{k-1} e_{k}
$$

we have that $E N_{k}\left(a_{1}, \ldots, a_{k-1}\right)$ is isomorphic to the algebra $E N_{k}$, which complete the proof of proposition.

Below we establish the isomorphism of evolution algebras of permutations with given conjugated permutations.

Theorem 3.7. If permutations $\pi_{1}, \pi_{2} \in S_{n}$ are conjugated, then evolution algebras $E_{n, \pi_{1}}\left(a_{1}, \ldots, a_{n}\right)$ and $E_{n, \pi_{2}}\left(a_{1}, \ldots, a_{n}\right)$ are isomorphic.

Proof. Let $\pi_{1}, \pi_{2} \in S_{n}$ are conjugated, then there exists $g \in S_{n}$ such that $g \pi_{1}=\pi_{2} g$. The map $f: E_{n, \pi_{1}} \rightarrow E_{n, \pi_{2}}$ defined by $f\left(e_{i}\right)=e_{g(i)}$ is isomorphism. Indeed,

$$
a_{i} e_{g \pi_{1}(i)}=f\left(a_{i} e_{\pi_{1}(i)}\right)=f\left(e_{i} \cdot e_{i}\right)=f\left(e_{i}\right) \cdot f\left(e_{i}\right)=a_{i} e_{g(i)} * e_{g(i)}=a_{i} e_{\pi_{2} g(i)} .
$$


Thus, for an algebra $E_{n, \pi}\left(a_{1}, \ldots, a_{n}\right)$ we can always assume that $\pi=(1,2, \ldots, n)$ and table of multiplication is

where $a_{i} \in\{0 ; 1\}$.

$$
\begin{cases}e_{i} \cdot e_{i}=a_{i} e_{i+1}, & 1 \leq i \leq n-1, \\ e_{n} \cdot e_{n}=a_{n} e_{1}, & \\ e_{i} \cdot e_{j}=0, & i \neq j,\end{cases}
$$

Proposition 3.8. An arbitrary evolution algebra $E_{n, \pi}\left(a_{1}, a_{2}, \ldots, a_{n}\right)$ with $\pi=(1,2, \ldots, n)$ is isomorphic to the algebra $E_{n}$ or the direct sum of evolution algebras $E N_{k_{1}} \oplus E N_{k_{2}} \oplus \cdots \oplus E N_{k_{r}}$.

Proof. If all $a_{i} \neq 0$, then due to Theorem 3.5 we have that algebra $E_{n, \pi}\left(a_{1}, a_{2}, \ldots, a_{n}\right)$ is isomorphic to the $E_{n}$.

Let $a_{k}=0$ for some $k$ and $a_{i} \neq 0$ for $i \neq k$. Taking the basis transformation in the following form:

$$
\begin{gathered}
e_{1}^{\prime}=A_{1} e_{k+1}, e_{2}^{\prime}=A_{2} e_{k+2}, \ldots, e_{n-k}^{\prime}=A_{n-k} e_{n}, \\
e_{n-k+1}^{\prime}=A_{n-k+1} e_{1}, e_{n-k+2}^{\prime}=A_{n-k+2} e_{2}, \ldots, e_{n}^{\prime}=A_{n} e_{k},
\end{gathered}
$$

where

$$
\begin{gathered}
A_{1}=1, \quad A_{2}=a_{k+1}, \quad A_{3}=a_{k+1}^{2} a_{k+2}, \quad \ldots, \quad A_{n-k+1}=a_{k+1}^{2^{n-k-1}} a_{k+2}^{2^{n-k-2}} \ldots a_{n}, \\
A_{n-k+2}=a_{k+1}^{2^{n-k}} a_{k+2}^{2^{n-k-1}} \ldots a_{n}^{2} a_{1}, \ldots, \quad \ldots, \quad A_{n}=a_{k+1}^{2^{n-2}} a_{k+2}^{2^{n-3}} \ldots a_{n}^{2^{k-1}} a_{1}^{2^{k-2}} a_{2}^{2^{k-3}} \ldots a_{k-1},
\end{gathered}
$$

we derive isomorphism between algebra $E_{n, \pi}\left(a_{1}, \ldots, a_{k-1}, 0, a_{k+1}, \ldots, a_{n}\right)$ and algebra $E N_{n}$.

Applying similar arguments, we can establish that for $(r+1)$-times of parameters $a_{i}$ are equal to zero an algebra $E_{n}\left(a_{1}, a_{2}, \ldots, a_{n}\right)$ is isomorphic to

$$
E N_{k_{1}} \oplus E N_{k_{2}} \oplus \cdots \oplus E N_{k_{r}} .
$$

We resume the above results in the main theorem of this section.

Theorem 3.9. An arbitrary evolution algebra of permutations $E_{n, \pi}\left(a_{1}, a_{2}, \ldots, a_{n}\right)$ is isomorphic to a direct sum of algebras $E_{p_{1}}, E_{p_{2}}, \ldots, E_{p_{s}}, E N_{k_{1}}, E N_{k_{2}}, \ldots, E N_{k_{r}}$, i.e.,

$$
E_{n, \pi}\left(a_{1}, a_{2}, \ldots, a_{n}\right) \cong E_{p_{1}} \oplus E_{p_{2}} \oplus \cdots \oplus E_{p_{s}} \oplus E N_{k_{1}} \oplus E N_{k_{2}} \oplus \cdots \oplus E N_{k_{r}}
$$

In the description of evolution algebras of permutations from above theorem we get the importance of algebras $E_{n}, E N_{n}$, idempotents and absolute nilpotent elements.

\section{Associative enveloping algebras of some evolution algebras}

For a complex two-dimensional evolution algebra $E$ of the list of Theorem 2.2 we describe its associative enveloping algebra $M(E)$ :

$$
\begin{aligned}
& M\left(E_{1}\right)=\operatorname{alg}\left\langle R_{e_{1}}=\left(\begin{array}{ll}
1 & 0 \\
0 & 0
\end{array}\right)\right\rangle \cong x x=x ; \\
& M\left(E_{2}\right)=\operatorname{alg}\left\langle R_{e_{1}}=\left(\begin{array}{ll}
1 & 0 \\
0 & 0
\end{array}\right), R_{e_{2}}=\left(\begin{array}{cc}
0 & 0 \\
1 & 0
\end{array}\right)\right\rangle \cong x x=x, y x=x ; \\
& M\left(E_{3}\right)=\operatorname{alg}\left\langle R_{e_{1}}=\left(\begin{array}{ll}
1 & 1 \\
0 & 0
\end{array}\right), R_{e_{2}}=\left(\begin{array}{cc}
0 & 0 \\
-1 & -1
\end{array}\right)\right\rangle \cong x x=x, x y=-x, y x=y, y y=-y ; \\
& M\left(E_{4}\right)=\operatorname{alg}\left\langle R_{e_{1}}=\left(\begin{array}{ll}
0 & 1 \\
0 & 0
\end{array}\right)\right\rangle \cong x x=0 ; \\
& M\left(E_{5}(0,0)\right)=a l g\left\langle R_{e_{1}}=\left(\begin{array}{ll}
1 & 0 \\
0 & 0
\end{array}\right), R_{e_{2}}=\left(\begin{array}{cc}
0 & 0 \\
0 & 1
\end{array}\right)\right\rangle \cong x x=x, y y=y ; \\
& M\left(E_{5}\left(a_{2}, a_{3}\right)\right)\left(a_{2}=0 \text { or } a_{3}=0\right) \cong\left\{P \in M_{2}(\mathbb{C}) \mid P=\left(\begin{array}{cc}
b_{1} & b_{2} \\
0 & b_{3}
\end{array}\right)\right\}
\end{aligned}
$$




$$
\begin{aligned}
& M\left(E_{5}\left(a_{2}, a_{3}\right)\right)\left(a_{2} a_{3} \neq 0\right)=\operatorname{alg}\left\langle R_{e_{1}}=\left(\begin{array}{cc}
1 & a_{2} \\
0 & 0
\end{array}\right), R_{e_{2}}=\left(\begin{array}{cc}
0 & 0 \\
a_{3} & 1
\end{array}\right)\right\rangle \cong M_{2}(\mathbb{C}) ; \\
& M\left(E_{6}\left(a_{4}\right)\right)=\operatorname{alg}\left\langle R_{e_{1}}=\left(\begin{array}{ll}
0 & 1 \\
0 & 0
\end{array}\right), R_{e_{2}}=\left(\begin{array}{cc}
0 & 0 \\
1 & a_{4}
\end{array}\right)\right\rangle \cong M_{2}(\mathbb{C}) .
\end{aligned}
$$

Take the element $x=\sum_{i=1}^{n} x_{i} e_{i}$. Let $x \cdot x=0$, then we have

$$
x \cdot x=\left(\sum_{i=1}^{n} x_{i} e_{i}\right)\left(\sum_{i=1}^{n} x_{i} e_{i}\right)=\sum_{i=1}^{n} x_{i}^{2} \sum_{j=1}^{n} a_{i, j} e_{j}=\sum_{j=1}^{n}\left(\sum_{i=1}^{n} x_{i}^{2} a_{i, j}\right) e_{j} .
$$

From this we have

$$
\left\{\begin{array}{l}
a_{1,1} x_{1}^{2}+a_{2,1} x_{2}^{2}+\cdots+a_{n, 1} x_{n}^{2}=0 \\
a_{1,2} x_{1}^{2}+a_{2,2} x_{2}^{2}+\cdots+a_{n, 2} x_{n}^{2}=0 \\
\cdots \cdots \cdots \cdots \cdots \cdots \cdots \cdots \cdots \cdots+\cdots \cdots+a_{n, n} x_{n}^{2}=0 \\
a_{1, n} x_{1}^{2}+a_{2, n} x_{2}^{2}+\cdots \cdots
\end{array}\right.
$$

This system has a non-trivial solution if and only if $\operatorname{det} A^{t}=\operatorname{det} A=0$.

Note that for the case of Markov evolution algebra, by summing above equalities, we conclude $x_{1}^{2}+x_{2}^{2}+\cdots+x_{n}^{2}=0$. If Markov evolution algebra is real, then $x_{i}=0,1 \leq i \leq n$, that is, $x=0$.

Thus we proved the following proposition.

Proposition 4.1. A complex evolution algebra has non-trivial absolute nilpotent elements if and only if $\operatorname{det} A=0$. Moreover, if real evolution algebra is Markov, then it has only trivial absolute nilpotent elements.

For an $n$-dimensional evolution algebra $E$ we consider an associative enveloping algebra $M(E)$. Due to linearity the right multiplication operator one gets $M(E)=$ alg < $R_{e_{i}} \mid e_{i}$ is a basis element of $E>$.

Using the equalities $R_{e_{i}}\left(e_{i}\right)=\sum_{k=1}^{n} a_{i, k} e_{k}$ and $R_{e_{i}}\left(e_{j}\right)=0$ for $i \neq j$, we obtain a matrix form of $R_{e_{i}}$ as follows:

$$
R_{e_{i}}=\sum_{k=1}^{n} a_{i, k} e_{i, k}
$$

where $e_{i, k}$ are matrix units.

One can get

$$
R_{e_{i}} R_{e_{j}}=a_{i, j} \sum_{k=1}^{n} a_{j, k} e_{i, k} .
$$

From (4.1) we conclude that $\operatorname{dim} M(E)=r_{1}+r_{2}+\cdots+r_{n}$ with

$$
r_{i}=\operatorname{rank}\left(\begin{array}{cccc}
a_{i, 1} a_{1,1} & a_{i, 1} a_{1,2} & \ldots & a_{i, 1} a_{1, n} \\
a_{i, 2} a_{2,1} & a_{i, 2} a_{2,2} & \ldots & a_{i, 2} a_{2, n} \\
\vdots & \vdots & \vdots & \vdots \\
a_{i, n} a_{n, 1} & a_{i, n} a_{n, 2} & \ldots & a_{i, n} a_{n, n}
\end{array}\right) .
$$

Further we shall consider some cases for $n$-dimensional evolution algebra $E$ with structural constant matrix $A$ and satisfying the condition: $\operatorname{dim} M(E)=n$.

Proposition 4.2. Let $\operatorname{rank} A=1$. Then associative enveloping algebra $M(E)$ is isomorphic to one of the following algebras:

$$
M^{s}: x_{i} x_{j}=x_{i}, \quad 1 \leq i \leq s, 1 \leq j \leq n .
$$

Proof. Due to conditions of proposition we have the table of multiplication of algebra $E$ :

$$
e_{i} \cdot e_{i}=t_{i} \sum_{k=1}^{n} a_{k} e_{k}
$$


The condition $\operatorname{dim} M(E)=n$ implies $t_{i} \neq 0$ for any $i(1 \leq i \leq n)$ and basis of $M(E)$ can be chosen as $\left\{R_{e_{1}}, R_{e_{2}}, \ldots, R_{e_{n}}\right\}$, where $R_{e_{i}}=t_{i} \sum_{k=1}^{n} a_{k} e_{i, k}$. Therefore, the table of multiplication of the algebra $M(E)$ has the form:

$$
R_{e_{i}} R_{e_{j}}=t_{j} a_{j} R_{e_{i}}, 1 \leq i, j \leq n .
$$

By appropriate shifting of basis elements, without loss of generality, one can assume $a_{i} \neq 0$ for $1 \leq i \leq s, s \leq n$ and $a_{j}=0$ for $s+1 \leq j \leq n$. The scaling the basis elements $e_{i}, 1 \leq i \leq s$ reduces our study to the case of $t_{i} a_{i}=1$. Thus, we obtain the tables of multiplication of associative enveloping algebras $M^{s}$.

Below we present the description of $n$-dimensional associative enveloping algebras $M(E)$ for evolution algebra $E$ with $\operatorname{rank} A=n$.

Proposition 4.3. Let $\operatorname{rank} A=n$. Then associative enveloping algebra $M(E)$ is isomorphic to the algebra

$$
M_{1}: \quad x_{i} x_{i}=x_{i}, \quad 1 \leq i \leq n .
$$

Proof. Taking into account in the equalities

$$
R_{e_{i}} R_{e_{j}}=a_{i, j} \sum_{k=1}^{n} a_{j, k} e_{i, k}=\beta_{1} R_{e_{1}}+\beta_{2} R_{e_{2}}+\cdots+\beta_{n} R_{e_{n}}
$$

that $\left\{R_{e_{1}}, R_{e_{2}}, \ldots, R_{e_{n}}\right\}$ are linear independent (they are forms a basis of $M(E)$ ), we conclude $\beta_{k}=0$ for $k \neq i$.

Therefore,

$$
a_{i, j} \sum_{k=1}^{n} a_{j, k} e_{i, k}=\beta_{i} R_{e_{i}}=\beta_{i} \sum_{k=1}^{n} a_{i, k} e_{i, k}
$$

From (4.2) we derive

$$
a_{i, j} a_{j, k}=\beta_{i} a_{i, k}, \quad 1 \leq k \leq n
$$

The condition $\operatorname{rank} A=n$, implies $\operatorname{rank}\left(\begin{array}{cccc}a_{i, 1} & a_{i, 2} & \ldots & a_{i, n} \\ a_{j, 1} & a_{j, 2} & \ldots & a_{j, n}\end{array}\right)=2$ for any $i \neq j$.

Using the arbitrariness $k$ in the equality (4.3) we obtain

$$
a_{i, j}=0, \quad i \neq j \quad \Rightarrow \quad a_{i, i} \neq 0 .
$$

Therefore,

$$
R_{e_{i}} R_{e_{i}}=a_{i, i} R_{e_{i}}, \quad 1 \leq i \leq n .
$$

By scaling the basis elements, we can suppose $a_{i, i}=1$ and the algebra $M_{1}$ is obtained.

The list of $n$-dimensional algebras $M(E)$ for an evolution algebra, satisfying the condition $\operatorname{rank} A=$ $n-1$, is presented in the following theorem.

Theorem 4.4. Let $\operatorname{rank} A=n-1$. Then associative enveloping algebra $M(E)$ is isomorphic to one of the following algebras:

$$
\begin{gathered}
M_{2}: \quad x_{i} x_{i}=x_{i}, 1 \leq i \leq n, x_{1} x_{n}=x_{1}, x_{n} x_{1}=x_{n}, \\
M_{3}: \quad x_{i} x_{i}=x_{i}, 1 \leq i \leq n-1, x_{n} x_{1}=x_{n}, \\
M_{4}: \quad x_{i} x_{i}=x_{i}, 1 \leq i \leq n-1, \quad x_{1} x_{2}=x_{n}, \quad x_{1} x_{n}=x_{n}, \quad x_{n} x_{2}=x_{n} .
\end{gathered}
$$

Proof. Without loss of generality, one can assume

$$
A=\left(\begin{array}{cccc}
a_{1,1} & a_{1,2} & \ldots & a_{1, n} \\
a_{2,1} & a_{2,2} & \ldots & a_{2, n} \\
\vdots & \vdots & \vdots & \vdots \\
a_{n-1,1} & a_{n-1,2} & \ldots & a_{n-1, n} \\
\sum_{i=1}^{n-1} \alpha_{i} a_{i, 1} & \sum_{i=1}^{n-1} \alpha_{i} a_{i, 2} & \ldots & \sum_{i=1}^{n-1} \alpha_{i} a_{i, n}
\end{array}\right) .
$$


Case 1. Let $R_{e_{n}}=\sum_{k=1}^{n}\left(\sum_{i=1}^{n-1} \alpha_{i} a_{i, k}\right) e_{n, k} \neq 0$. Then $\left\{R_{e_{1}}, R_{e_{1}}, \ldots, R_{e_{n-1}}, R_{e_{n}}\right\}$ is a basis of $M(E)$. Similarly as in the proof of Proposition 4.3, from the condition

$$
\operatorname{rank}\left(\begin{array}{cccc}
a_{i, 1} & a_{i, 2} & \ldots & a_{i, n} \\
a_{j, 1} & a_{j, 2} & \ldots & a_{j, n}
\end{array}\right)=2,
$$

for any $1 \leq i, j \leq n-1, i \neq j$, we deduce

$$
a_{i, j}=0, \quad 1 \leq i, j \leq n-1, i \neq j .
$$

Consequently,

$$
R_{e_{i}}=a_{i, i} e_{i, i}+a_{i, n} e_{i, n}, 1 \leq i \leq n-1
$$

and

$$
R_{e_{n}}=\sum_{k=1}^{n-1} \alpha_{k} a_{k, k} e_{n, k}+\left(\sum_{s=1}^{n-1} \alpha_{s} a_{s, n}\right) e_{n, n} .
$$

If $\alpha_{k} a_{k, k} \neq 0$ for some $k(1 \leq k \leq n-1)$, then without loss of generality we can suppose $\alpha_{1} a_{1,1} \neq 0$. Consider

$$
R_{e_{n}} R_{e_{1}}=\alpha_{1} a_{1,1}^{2} e_{n, 1}+\alpha_{1} a_{1,1} a_{1, n} e_{n, n} .
$$

Since the product $R_{e_{n}} R_{e_{i}}$ should be expressed by $R_{e_{n}}$, we conclude $\alpha_{k} a_{k, k}=\alpha_{k} a_{k, n}=0,2 \leq k \leq n-1$, which yield

$$
\alpha_{k}=0, \quad 2 \leq k \leq n-1
$$

If $\alpha_{k} a_{k, k}=0$ for any $k(1 \leq k \leq n-1)$, then from the condition $R_{e_{n}} \neq 0$ we have the existence some $k_{0}$ such that $\alpha_{k_{0}} \neq 0$. Hence, $a_{k_{0}, k_{0}}=0$. Since $\operatorname{rank} A=n-1$, then $a_{k, k} \neq 0$ for any $k \neq k_{0}$, consequently, $\alpha_{k}=0$ for any $k \neq k_{0}$. Without loss of generality, one can assume $k_{0}=1$.

Thus, in Case 1 we obtain

$$
\alpha_{k}=0,2 \leq k \leq n-1 \text {. }
$$

Therefore,

Consider

$$
R_{e_{n}} R_{e_{i}}=0,2 \leq i \leq n-1, \quad R_{e_{1}} R_{e_{n}}=a_{1, n} R_{e_{1}} .
$$

$$
R_{e_{i}} R_{e_{n}}=\left(a_{i, i} e_{i, i}+a_{i, n} e_{i, n}\right)\left(\alpha_{1} a_{1,1} e_{n, 1}+\alpha_{1} a_{1, n} e_{n, n}\right)=a_{i, n}\left(\alpha_{1} a_{1,1} e_{i, 1}+\alpha_{1} a_{i, n} e_{i, n}\right) .
$$

Since $R_{e_{i}} R_{e_{n}}$ should belong to $<R_{e_{i}}>$, we get $a_{i, n}=0, \quad 2 \leq i \leq n-1$.

Thus, the table of multiplication of the algebra $M(E)$ has the following form:

$$
\begin{gathered}
R_{e_{i}} R_{e_{i}}=a_{i, i} R_{e_{i}} \quad 1 \leq i \leq n-1 \\
R_{e_{1}} R_{e_{n}}=a_{1, n} R_{e_{1}}, \quad R_{e_{n}} R_{e_{1}}=a_{1,1} R_{e_{n}}, \quad R_{e_{n}} R_{e_{n}}=a_{1, n} R_{e_{n}},
\end{gathered}
$$

where $\left(a_{1,1}, a_{1, n}\right) \neq(0,0)$ and $a_{i, i} \neq 0,2 \leq i \leq n$.

Considering the possible cases: $a_{1,1} a_{1, n} \neq 0$ and $a_{1,1} a_{1, n}=0$, one finds the algebras $M_{2}, M_{3}$.

Case 2. Let $R_{e_{n}}=0$. Since a non-zero products of the form $R_{e_{i}} R_{e_{j}}=a_{i, j} \sum_{k=1}^{n} a_{j, k} e_{i, k}$ are linear independent, we obtain the existence of a unique non-zero coefficient $a_{i_{0}, j_{0}}, 1 \leq i_{0}, j_{0} \leq n-1, i_{0} \neq j_{0}$. Without loss of generality, we can suppose $i_{0}=1, j_{0}=2$, i.e. $a_{1,2} \neq 0$.

Therefore,

$$
\begin{gathered}
a_{i, j}=0, \quad 1 \leq i, j \leq n-1, \quad i \neq j, \quad(i, j) \neq(1,2) . \\
a_{i, i} \neq 0, \quad 1 \leq i \leq n-1,
\end{gathered}
$$

Putting $x_{i}=R_{e_{i}}, 1 \leq i \leq n-1$ and $x_{n}=a_{2,2} e_{1,2}+a_{2, n} e_{1, n}$, we obtain the table of multiplications of the algebra $M(E)$ in the form:

$$
x_{i} x_{i}=a_{i, i} x_{i}, 1 \leq i \leq n-1, \quad x_{1} x_{2}=a_{1,2} x_{n}, \quad x_{1} x_{n}=a_{1,1} x_{n}, \quad x_{n} x_{2}=a_{2,2} x_{n} .
$$

Taking the change

$$
x_{1}^{\prime}=\frac{1}{a_{i, i}} x_{i}, 1 \leq i \leq n-1, \quad x_{n}^{\prime}=\frac{a_{1,2}}{a_{1,1} a_{2,2}} x_{n}
$$

we get the algebra $M_{4}$. 


\section{Three-dimensional evolution algebras Whose generators have infinite PeRiod.}

In this section we study three-dimensional evolution algebras whose generators have infinite period. Let $E$ be a three-dimensional evolution algebra $E$ with table of multiplications:

$$
e_{1} \cdot e_{1}=a_{1} e_{1}+a_{2} e_{2}+a_{3} e_{3}, \quad e_{2} \cdot e_{2}=b_{1} e_{1}+b_{2} e_{2}+b_{3} e_{3}, \quad e_{3} \cdot e_{3}=c_{1} e_{1}+c_{2} e_{2}+c_{3} e_{3} .
$$

Since the periods of generators are infinite, we get $a_{1}=b_{2}=c_{3}=0$.

Consider $e_{i}^{[3]}$ and $e_{i}^{[4]}$ for $1 \leq i \leq 3$

$$
\begin{gathered}
\left\{\begin{array}{l}
e_{1}^{[3]}=\left(a_{2}^{2} b_{1}+a_{3}^{2} c_{1}\right) e_{1}+a_{3}^{2} c_{2} e_{2}+a_{2}^{2} b_{3} e_{3}, \\
e_{2}^{[3]}=b_{3}^{2} c_{1} e_{1}+\left(b_{1}^{2} a_{2}+b_{3}^{2} c_{2}\right) e_{2}+b_{1}^{2} a_{3} e_{3}, \\
e_{3}^{[3]}=c_{2}^{2} b_{1} e_{1}+c_{1}^{2} a_{2} e_{2}+\left(c_{1}^{2} a_{3}+c_{2}^{2} b_{3}\right) e_{3},
\end{array}\right. \\
\left\{\begin{array}{l}
e_{1}^{[4]}=\left(a_{3}^{4} c_{2}^{2} b_{1}+a_{2}^{4} b_{3}^{2} c_{1}\right) e_{1}+a_{2}^{4} b_{3}^{2} c_{2} e_{2}+a_{3}^{4} c_{2}^{2} b_{3} e_{3}, \\
e_{2}^{[4]}=b_{1}^{4} a_{3}^{2} c_{1} e_{1}+\left(b_{3}^{4} c_{1}^{2} a_{2}+b_{1}^{4} a_{3}^{2} c_{2}\right) e_{2}+b_{3}^{4} c_{1}^{2} a_{3} e_{3}, \\
e_{3}^{[4]}=c_{1}^{4} a_{2}^{2} b_{1} e_{1}+c_{2}^{4} b_{1}^{2} a_{2} e_{2}+\left(c_{2}^{4} b_{1}^{2} a_{3}+c_{1}^{4} a_{2}^{2} b_{3}\right) e_{3} .
\end{array}\right.
\end{gathered}
$$

Taking account the condition on periods of generators, we derive

$$
\begin{gathered}
a_{2}^{2} b_{1}+a_{3}^{2} c_{1}=0, \quad b_{1}^{2} a_{2}+b_{3}^{2} c_{2}=0, \quad c_{1}^{2} a_{3}+c_{2}^{2} b_{3}=0, \\
a_{3}^{4} c_{2}^{2} b_{1}+a_{2}^{4} b_{3}^{2} c_{1}=0, \quad b_{3}^{4} c_{1}^{2} a_{2}+b_{1}^{4} a_{3}^{2} c_{2}=0, \quad c_{2}^{4} b_{1}^{2} a_{3}+c_{1}^{4} a_{2}^{2} b_{3}=0 .
\end{gathered}
$$

Theorem 5.1. Let $E$ be a three-dimensional evolution algebra with the table of multiplication (5.1). Let any basis element has an infinite period and $a_{2} a_{3} b_{1} b_{3} c_{1} c_{2}=0$. Then $E$ is isomorphic to the following evolution algebra:

$$
E^{1}: e_{1} \cdot e_{1}=a_{2} e_{2}+a_{3} e_{3}, \quad e_{2} \cdot e_{2}=b_{3} e_{3} .
$$

Proof. Let $a_{2} a_{3} b_{1} b_{3} c_{1} c_{2}=0$, then, without loss of generality, we can assume $b_{1}=0$. The equalities (5.2) and (5.3) imply

$$
a_{3} c_{1}=0, \quad b_{3} c_{2}=0, \quad a_{2} b_{3} c_{1}=0 .
$$

Case 1. Let $a_{3}=b_{3}=0$. Then we obtain products

$$
e_{1} \cdot e_{1}=a_{2} e_{2}, \quad e_{3} \cdot e_{3}=c_{1} e_{1}+c_{2} e_{2} .
$$
$E^{1}$.

Taking the change $e_{1}^{\prime}=e_{3}, e_{2}^{\prime}=e_{1}, e_{3}^{\prime}=e_{2}$, we derive that this algebra is isomorphic to the algebra

Case 2. Let $a_{3}=0$ and $b_{3} \neq 0$. Then from (5.4) we have $c_{2}=a_{2} c_{1}=0$.

If $a_{2} \neq 0$, then $c_{1}=0$ and we obtain evolution algebra with multiplications:

$$
e_{1} \cdot e_{1}=a_{2} e_{2}, e_{2} \cdot e_{2}=b_{3} e_{3} .
$$

If $a_{2}=0$, then the table of multiplications of the algebra $E$ is as follows:

$$
e_{2} \cdot e_{2}=b_{3} e_{3}, e_{3} \cdot e_{3}=c_{1} e_{1} .
$$

Putting $e_{1}^{\prime}=e_{2}, e_{2}^{\prime}=e_{3}, e_{3}^{\prime}=e_{1}$, we derive that this algebra is isomorphic to the algebra $E^{1}$.

Case 3. Let $a_{3} \neq 0$. Then restrictions (5.4) imply $c_{1}=b_{3} c_{2}=0$.

If $b_{3} \neq 0$, then $c_{2}=0$ and the algebra $E^{1}$ is obtained.

If $b_{3}=0$, then by taking basis transformation as follows:

$$
e_{1}^{\prime}=e_{1}, e_{2}^{\prime}=e_{3}, e_{3}^{\prime}=e_{2}
$$

we get $E^{1}$.

Theorem 5.2. Let $E$ be a three-dimensional evolution algebra with the table of multiplication (5.1) and let $a_{2} a_{3} b_{1} b_{3} c_{1} c_{2} \neq 0$. Then period of each basis elements of the algebra $E$ is infinite if and only if equations (5.2) hold true. 
Proof. It is sufficient to proof the part Only if. From equalities (5.2) we get

$$
a_{2}=-\frac{b_{3}^{2} c_{2}}{b_{1}^{2}}, \quad a_{3}=-\frac{c_{2}^{2} b_{3}}{c_{1}^{2}} .
$$

Putting this restrictions to the equality $a_{2}^{2} b_{1}+a_{3}^{2} c_{1}=0$ we obtain

$$
0=a_{2}^{2} b_{1}+a_{3}^{2} c_{1}=\frac{b_{3}^{2} c_{2}^{2}\left(b_{3}^{2} c_{1}^{3}+b_{1}^{3} c_{2}^{2}\right)}{b_{1}^{3} c_{1}^{3}},
$$

which implies

$$
b_{3}^{2} c_{1}^{3}+b_{1}^{3} c_{2}^{2}=0
$$

Similarly, we derive

$$
\begin{aligned}
& b_{1}=-\frac{a_{3}^{2} c_{1}}{a_{2}^{2}}, \quad b_{3}=-\frac{c_{1}^{2} a_{3}}{c_{2}^{2}} \quad \Rightarrow \quad a_{3}^{2} c_{2}^{3}+a_{2}^{3} c_{1}^{2}=0, \\
& c_{1}=-\frac{a_{2}^{2} b_{1}}{a_{3}^{2}}, \quad c_{2}=-\frac{b_{1}^{2} a_{2}}{b_{3}^{2}} \quad \Rightarrow \quad a_{2}^{2} b_{3}^{3}+a_{3}^{3} b_{1}^{2}=0 .
\end{aligned}
$$

Applying induction we will prove the following:

$$
e_{1}^{[k]}=A_{k, 2} e_{2}+A_{k, 3} e_{3}, \quad e_{2}^{[k]}=B_{k, 1} e_{1}+B_{k, 3} e_{3}, \quad e_{3}^{[k]}=C_{k, 1} e_{1}+C_{k, 2} e_{2}, k \geq 3
$$

with recurrence expressions

$$
\begin{aligned}
& A_{k, 2}=A_{k-1,3}^{2} c_{2}, \quad A_{k, 3}=A_{k-1,2}^{2} b_{3}, \quad A_{k-1,2}^{2} b_{1}+A_{k-1,3}^{2} c_{1}=0, \\
& B_{k, 1}=B_{k-1,3}^{2} c_{1}, \quad B_{k, 3}=B_{k-1,1}^{2} a_{3}, \quad B_{k-1,1}^{2} a_{2}+B_{k-1,3}^{2} c_{2}=0, \\
& C_{k, 1}=C_{k-1,2}^{2} b_{1}, \quad C_{k, 2}=C_{k-1,1}^{2} a_{2}, \quad C_{k-1,1}^{2} a_{3}+C_{k-1,3}^{2} b_{3}=0 \text {, }
\end{aligned}
$$

where $A_{2,2}=a_{2}, A_{2,3}=a_{3}, B_{2,1}=b_{1}, B_{2,3}=b_{3}, C_{2,1}=c_{1}, C_{2,2}=c_{2}$.

In fact, the correctness of expressions (5.8) is equivalent to that each basis element of evolution algebra $E$ has infinite period.

From decompositions of $e_{i}^{[3]}, 1 \leq i \leq 3$ it is easy to see the correctness of (5.9) for $k=3$, i.e.

$$
\begin{array}{lll}
A_{3,2}=a_{3}^{2} c_{2}=A_{2,3}^{2} c_{2}, & A_{3,3}=a_{2}^{2} b_{3}=A_{2,2}^{2} b_{3}, & A_{2,2}^{2} b_{1}+A_{2,3}^{2} c_{1}=a_{2}^{2} b_{1}+a_{3}^{2} c_{1}=0, \\
B_{3,1}=b_{3}^{2} c_{1}=B_{2,3}^{2} c_{1}, & B_{3,3}=b_{1}^{2} a_{3}=B_{2,1}^{2} a_{3}, & B_{2,1}^{2} a_{2}+B_{2,3}^{2} c_{2}=b_{1}^{2} a_{2}+b_{3}^{2} c_{2}=0, \\
C_{3,1}=c_{2}^{2} b_{1}=C_{2,2}^{2} b_{1}, & C_{3,2}=c_{1}^{2} a_{2}=C_{2,1}^{2} a_{2}, & C_{2,1}^{2} a_{3}+C_{2,2}^{2} b_{3}=c_{1}^{2} a_{3}+c_{2}^{2} b_{3}=0 .
\end{array}
$$

Suppose that (5.8) and (5.9) are true for $k$. We will prove it for $k+1$.

The chain of equalities

$e_{1}^{[k+1]}=e_{1}^{[k]} \cdot e_{1}^{[k]}=\left(A_{k, 2} e_{2}+A_{k, 3} e_{3}\right) \cdot\left(A_{k, 2} e_{2}+A_{k, 3} e_{3}\right)=\left(A_{k, 2}^{2} b_{1}+A_{k, 3}^{2} c_{1}\right) e_{1}+A_{k, 3}^{2} c_{2} e_{2}+A_{k, 2}^{2} b_{3} e_{3}$ deduces

$$
A_{k+1,2}=A_{k, 3}^{2} c_{2}, \quad A_{k+1,3}=A_{k, 2}^{2} b_{3}
$$

Applying induction assumption, that is,

$$
A_{k, 2}=A_{k-1,3}^{2} c_{2}, \quad A_{k, 3}=A_{k-1,2}^{2} b_{3}, \quad A_{k-1,2}^{2} b_{1}+A_{k-1,3}^{2} c_{1}=0,
$$

we get

$$
A_{k, 2}^{2} b_{1}+A_{k, 3}^{2} c_{1}=A_{k-1,3}^{4} c_{2}^{2} b_{1}+A_{k-1,2}^{4} b_{3}^{2} c_{1}=\frac{A_{k-1,2}^{4} b_{1}^{2}}{c_{1}^{2}} c_{2}^{2} b_{1}+A_{k-1,2}^{4} b_{3}^{2} c_{1}=\frac{A_{k-1,2}^{4}}{c_{1}^{2}}\left(b_{1}^{3} c_{2}^{2}+b_{3}^{2} c_{1}^{3}\right)
$$

The equality (5.5) implies $A_{k, 2}^{2} b_{1}+A_{k, 3}^{2} c_{1}=0$.

Similarly, one finds

$$
B_{k, 1}^{2} a_{2}+B_{k, 3}^{2} c_{2}=0, \quad C_{k, 1}^{2} a_{3}+C_{k, 2}^{2} b_{3}=0 .
$$

\section{ACKNOWLEDGEMENTS}

This works is supported by the Grant No.0251/GF3 of Education and Science Ministry of Republic of Kazakhstan and the Grant (RGA) No:11-018 RG/Math/AS_I-UNESCO FR: 3240262715. 


\section{REFERENCES}

[1] Camacho L.M., Gómez J.R., Omirov B.A., Turdibaev R.M. Some properties of evolution algebras, Bulletin of Korean Mathematical Society (to appear), arXiv:1004.1987 1.

[2] Camacho L.M., Gómez J.R., Omirov B.A., Turdibaev R.M. The derivations of some evolution algebras, Linear and Multilinear Algebra. doi: 10.1080/03081087.2012.678342.

[3] Casas J.M., Ladra M., Omirov B.A., Rozikov U.A. On nilpotent index and dibaricity of evolution algebras, Linear Algebra Appl., vol. 439, 2013, p. 90-105.

[4] Casas J.M., Ladra M., Omirov B.A., Rozikov U.A. On evolution algebras, Algebra Colloquium (to apperar), arXiv:1004.1050 1.

[5] Casas J. M., Ladra M., Rozikov U. A. Chain of evolution algebras, Linear Algebra Appl., vol. 435, 2011, no. 4, pp. $852-870$.

[6] Ladra M., Omirov B.A., Rozikov U.A. On dibaric and evolution algebras, arXiv:1104.2578v1.

[7] Lyubich Y.I. Mathematical structures in population genetics, Springer-Verlag, Berlin, 1992.

[8] Reed M.L. Algebraic structure of genetic inheritance, Bull. Amer. Math. Soc. (N.S.) 34(2) (1997), 107130.

[9] Rozikov U.A., Tian J.P. Evolution algebras generated by Gibbs measures, Lobachevskii Jour. Math. 32 (4) (2011) $270-277$.

[10] Tian J.P. Evolution algebras and their applications, Lecture Notes in Math., 1921. Springer, Berlin, 2008.

[11] Wörz-Busekros A. Algebras in genetics, Lecture Notes in Biomathematics 36, Springer-Verlag, Berlin-New York, 1980.

[A. Kh. Khudoyberdiyev and B. A. Omirov] Institute of Mathematics, National University of Uzbekistan, TASHKEnt, 100125, UzBekistan.

E-mail address: khabror@mail.ru, omirovb@mail.ru

[Izzat Qaralleh] I Department of Computational \& Theoretical Sciences, Faculty of Science, International Islamic University Malaysia, P.O. Box, 141, 25710, Kuantan, Pahang, Malaysia

E-mail address: izzat_math@yahoo.com 